\title{
3D MULTIZONE SOUNDFIELD REPRODUCTION IN A REVERBERANT ENVIRONMENT USING INTENSITY MATCHING METHOD
}

\author{
Huanyu Zuo, Thushara D. Abhayapala, Prasanga N. Samarasinghe
}

\author{
Audio \& Acoustic Signal Processing Group, The Australian National University, Canberra, Australia
}

\begin{abstract}
Sound intensity is a good predictor of human perception of sound location, which can be controlled to provide impressive direction perception to humans in soundfield reproduction systems, especially when the loudspeakers are non-uniformly distributed. However, the previous works in this field are all constrained to a single sweet spot/spatial zone. We address this challenge and propose a multizone reproduction method for $3 \mathrm{D}$ soundfield in a reverberant room based on intensity matching. We develop spatial sound intensity expressions in a reververant room using spherical harmonic decomposition, and build a cost function to optimize sound intensity within multiple spatial zones. Finally, simulation results showing the performance are presented.
\end{abstract}

Index Terms - Multizone, soundfield reproduction, sound intensity, spherical harmonics, human perception

\section{INTRODUCTION}

Spatial multizone soundfield reproduction aims to reproduce desired soundfields over multiple spatial regions, which has various applications such as simultaneous entertainment systems in cars and personal audio systems in shared office spaces. To achieve such personal sound zones, the multizone reproduction was firstly formulated as creating a bright zone and a dark zone by maximizing the ratio of energy in the two zones, which is known as the acoustic contrast control method $[1,2,3]$. Since then, different approaches based on the acoustic contrast control have been proposed, including the pressure matching approach $[4,5,6,7]$, and the modaldomain approach $[8,9,10,11]$. Besides of creating such two kinds of sound zones (i.e., the bright zone and dark zone), the generation of two zones of silence was investigated in [12]. In [13], the authors recreated two active sound zones by translating the local soundfields to an equivalent global soundfield and then reconstructing the equivalent soundfield using mode matching approach. However, these works are based on sound pressure optimization, which is not directly linked with human perception. This may lead to percep-

This work is supported by ARC Linkage Grant No. LP160100379 and the China Scholarship Council with the Australian National University. tion degradation when there are only a limited number of non-uniformly distributed loudspeakers [14].

Sound intensity has been controlled to improve reproduction performance in the perspective of perception since Gerzon developed the sound intensity theory of sound localization for reproducing psychoacoustically optimum sound [15]. In addition to good performance for uniformly distributed loudspeakers $[16,17]$, the intensity based reproduction methods can achieve good localization perception in spatially non-uniform loudspeaker arrangements [18, 19]. Besides, they can also provide less coloration (i.e., changes in the timbre) compared with the sound pressure based methods [20]. However, these intensity based works are all restricted to a single reproduction position, and therefore perception degrades when listeners are moved from this exact reproduction position. Recently, we proposed an intensity matching technique to optimally reproduce sound intensity over a continuous spatial region using a non-uniformly placed loudspeaker array [21], which allows listeners to freely move over the region with good perception.

In this paper, we extend [21] to multiple spatial zones, concurrently taking room acoustics into consideration. Our aim is to psychoacoustically create the impression of the desired sound in each spatial zone by controlling sound intensity within the zone. We derive sound intensity expressions in a reverberant room and formulate the multizone reproduction as an optimization problem based on sound intensity. Finally, we compare the proposed method with the conventional multizone reproduction method of mode matching in a spatially non-uniform loudspeaker arrangement through numerical simulations.

\section{PROBLEM FORMULATION}

We assume that there are a set of distinct 3D spherical zones in a reverberant room and corresponding desired spatial soundfields. As shown in Fig.1, the radius and the origin of the $b^{\text {th }}$ spherical zone are denoted as $R_{b}$ and $O_{b}$, respectively, where $O_{b}$ is located at the spherical coordinate $\left(r_{o}^{(b)}, \theta_{o}^{(b)}, \phi_{o}^{(b)}\right)$ with respect to the global origin $O$. Any arbitrary observation point within this $b^{\text {th }}$ spherical zone is denoted as $\boldsymbol{x}_{b}=\left(r_{b}, \theta_{b}, \phi_{b}\right)$ with respect to the corresponding local origin $O_{b}$. The loudspeakers are placed outside of a 


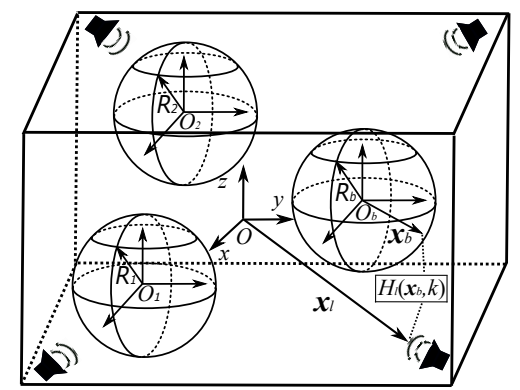

Fig. 1. Geometry of the multizone reproduction system in this paper. The loudspeaker array surrounds the zones.

sphere with radius of $R_{\max }$ from $O$ in the room, where $R_{\max }$ is the maximum value of $r_{o}^{(b)}+r_{b}$ for all the zones (i.e., the loudspeaker array encompasses all the spherical zones). The location of the $\ell^{\text {th }}$ loudspeaker is denoted as $\boldsymbol{x}_{\ell}=\left(r_{\ell}, \theta_{\ell}, \phi_{\ell}\right)$ with respect to $O$.

Given the desired spatial soundfield $\left\{\alpha_{n m}^{(b)}(k)\right\}$, where $\alpha_{n m}^{(b)}(k)$ are desired pressure coefficients ${ }^{1}$, for the $b^{\text {th }}$ spherical zone, the components of desired sound intensity $\boldsymbol{I}^{d}\left(\boldsymbol{x}_{b}, k\right)=$ $\left[I_{r}^{d}\left(\boldsymbol{x}_{b}, k\right), I_{\theta}^{d}\left(\boldsymbol{x}_{b}, k\right), I_{\phi}^{d}\left(\boldsymbol{x}_{b}, k\right)\right]$ at any arbitrary $\boldsymbol{x}_{b}$ within the zone can be written as [22,23]

$I_{\Psi}^{d}\left(\boldsymbol{x}_{b}, k\right)=\sum_{p=0}^{Q} \sum_{q=-p}^{p} S_{p q}^{(\Psi, d)}\left(k, r_{b}\right) Y_{p q}\left(\theta_{b}, \phi_{b}\right) ; \Psi \in\{r, \theta, \phi\}$,

with

$$
\begin{aligned}
& S_{p q}^{(r, d)}\left(k, r_{b}\right)=\frac{i}{k \rho_{0} c} \sum_{n=0}^{N} \sum_{m=-n}^{n} \sum_{n^{\prime}=0}^{N} \sum_{m^{\prime}=-n^{\prime}}^{n^{\prime}}(-1)^{m+q} \\
& \times C_{n n^{\prime} p} \alpha_{n m}^{(b) *}(k) \alpha_{n^{\prime} m^{\prime}}^{(b)}(k) j_{n}\left(k r_{b}\right) j_{n \prime}^{\prime}\left(k r_{b}\right) W_{1} W_{2},
\end{aligned}
$$

denoting spherical harmonic coefficients of desired sound intensity in $r$ direction, where

$$
C_{n n^{\prime} p}=\sqrt{(2 n+1)\left(2 n^{\prime}+1\right)(2 p+1) / 4 \pi},
$$

$j_{n}(\cdot)$ is the $n^{\text {th }}$ order spherical Bessel function of the first kind, $j_{n}^{\prime}(\cdot)$ is the derivative of $j_{n}(\cdot)$ in terms of $r, \rho_{0}$ is medium density, $N=\left\lceil k e R_{b} / 2\right\rceil$ is the truncation limit of the soundfield orders [24], $k=2 \pi f / c$ is the wave number, $f$ is the frequency, $c$ is the speed of propagation,

$$
W_{1}=\left(\begin{array}{ccc}
n & n^{\prime} & p \\
0 & 0 & 0
\end{array}\right) \text {, and } W_{2}=\left(\begin{array}{ccc}
n & n^{\prime} & p \\
-m & m^{\prime} & -q
\end{array}\right)
$$

are Wigner 3-j symbols [25], $Q=2 N$ is truncation order for intensity expressions [22], and $Y_{p q}(\theta, \phi)=A_{p q} P_{p q}(\cos \theta) e^{i q \phi}$ is the spherical harmonic of order $p$ and degree $q$ with $A_{p q}=[(2 p+1)(p-q) ! /(4 \pi(p+q) !)]^{1 / 2}$, where $P_{p q}(\cos \theta)$ are the associated Legendre functions. In this work, we select the same truncation order for all components of sound intensity for simplicity, at the cost of relatively more error on the component in $\theta$ and $\phi$ direction compared to $r$ direction.

\footnotetext{
${ }^{1} \mathrm{~A}$ soundfield in a spherical region is usually characterized by spherical harmonic coefficients of sound pressure, which can be extracted by using higher order microphones such as an EigenMike.
}

Both $S_{p q}^{(\theta, d)}\left(k, r_{b}\right)$ and $S_{p q}^{(\phi, d)}\left(k, r_{b}\right)$ have similar expressions to (2) and are given in our previous work [22,23].

The desired sound intensity distributions of all the spatial zones are available in the form of (1). Our objective is to design the driving functions for a loudspeaker array that will simultaneously reproduce all of those intensity distributions in multiple zones.

\section{SPATIAL SOUND INTENSITY REPRESENTATION IN A REVERBERANT ENVIRONMENT}

In a reverberant room, reflections should be taken into consideration in addition to the direct path. We present the pointto-region transfer function in this section, which incorporates both the direct path and reflections.

\subsection{Point-to-region transfer function}

With respect to $O_{b}$, the acoustic transfer function of the $\ell^{\text {th }}$ loudspeaker to $\boldsymbol{x}_{b}, H_{\ell}\left(\boldsymbol{x}_{b}, k\right)$, can be expressed by the spherical harmonic decomposition as

$$
H_{\ell}\left(\boldsymbol{x}_{b}, k\right)=\sum_{n=0}^{N} \sum_{m=-n}^{n} \beta_{n m}^{(\ell, b)}(k) j_{n}\left(k r_{b}\right) Y_{n m}\left(\theta_{b}, \phi_{b}\right),
$$

where $\beta_{n m}^{(\ell, b)}(k)$ are the sound field coefficients of the acoustic transfer function of the $\ell^{\text {th }}$ loudspeaker to the $b^{\text {th }}$ zone. Note that the position of the $\ell^{\text {th }}$ loudspeaker is $\boldsymbol{x}_{\ell}-\boldsymbol{O}_{b}$ with respect to $O_{b}$. We also call (3) point-to-region transfer function, because the acoustic transfer function of the $\ell^{\text {th }}$ loudspeaker to any point in the $b^{\text {th }}$ zone is readily available if $\beta_{n m}^{(\ell, b)}(k)$ are known.

In this paper, we simulate $\beta_{n m}^{(\ell, b)}(k)$ using the spherical harmonics based generalized image source method [26]. We assume all the loudspeakers as omni-directional point sources, and model the reverberant environment as a shoebox room with the size denoted by $\left(L_{x}, L_{y}, L_{z}\right)$ for length, width and height. Reverberant characteristics are modeled with the reflection coefficients of the wall surface, denoted as $\boldsymbol{d}=\left(d_{x 1}, d_{x 2}, d_{y 1}, d_{y 2}, d_{z 1}, d_{z 2}\right)$. The infinite image depth is truncated to $\boldsymbol{R}_{\text {depth }}$ for simplicity. Every single loudspeaker in the loudspeaker array is regarded as a separate source region. The coefficients of the point-to-region transfer function of the $\ell^{\text {th }}$ loudspeaker to the $b^{\text {th }}$ zone in the reverberant environment can be written as [26]

$$
\beta_{n m}^{(\ell, b)}(k)=\frac{i k}{\sqrt{4 \pi}} \alpha_{n m}^{00}(k),
$$

where $\alpha_{n m}^{00}(k)$ are the coupling coefficients. The exact expression of $\alpha_{n m}^{00}(k)$ and its detailed derivation are given in [26]. The location information of the $\ell^{\text {th }}$ loudspeaker and the $b^{\text {th }}$ zone, as well as the room parameters, is incorporated in $\alpha_{n m}^{00}(k)$.

Note that (4) holds only for the simulated shoebox room. For a real-world room with arbitrary geometries, one can estimate $\beta_{n m}^{(\ell, b)}(k)$ from room impulse response measurements. 


\subsection{Reproduced sound intensity}

The sound intensity for the $b^{\text {th }}$ zone due to the $\ell^{\text {th }}$ loudspeaker can be written, by replacing $\alpha_{n m}^{(b)}(k)$ with $\beta_{n m}^{(\ell, b)}(k)$ in $S_{p q}^{(\Psi, d)}\left(k, r_{b}\right)$ of (1), as

$$
I_{\Psi}^{(\ell)}\left(\boldsymbol{x}_{b}, k\right)=\sum_{p=0}^{Q} \sum_{q=-p}^{p} S_{p q}^{(\Psi, \ell)}\left(k, r_{b}\right) Y_{p q}\left(\theta_{b}, \phi_{b}\right),
$$

where $S_{p q}^{(\Psi, \ell)}\left(k, r_{b}\right)$ are intensity coefficients in $\Psi$ direction.

Applying a frequency-dependent weight $w_{\ell}(k)$ to the $\ell^{\text {th }}$ loudspeaker, the total sound intensity at $\boldsymbol{x}_{b}$ due to the loudspeaker array is given by

$$
I_{\Psi}^{a}\left(\boldsymbol{x}_{b}, k\right)=\sum_{\ell=0}^{N_{L}}\left|w_{\ell}(k)\right|^{2} I_{\Psi}^{(\ell)}\left(\boldsymbol{x}_{b}, k\right),
$$

where $N_{L}$ is the number of loudspeakers. Note that here we assume incoherent superposition of loudspeaker signals [21].

\section{MULTIZONE SOUNDFIELD REPRODUCTION}

The multizone reproduction is now reduced to calculate the loudspeaker weights that can reconstruct the desired sound intensity for multiple spatial zones. To accurately reconstruct the desired sound intensity within a bounded zone, it requires that the desired intensity coefficients on the surface of the zone (i.e., $\left.S_{p q}^{(\Psi, d)}\left(k, R_{b}\right)\right)$ are accurately reconstructed [21]. Therefore, the problem can be formulated mathematically as

$$
\min _{\boldsymbol{W}}\left\{\sum_{b=1}^{N_{P}} \tau_{b}^{2}\left\|\boldsymbol{S}_{A}\left(k, R_{b}\right) \boldsymbol{W}(k)-\boldsymbol{S}_{D}\left(k, R_{b}\right)\right\|_{2}^{2}\right\}, \text { s.t. } \boldsymbol{W} \geq 0,
$$

where $N_{P}$ is the number of the spatial zones, and $\tau_{b}$ is the weighting coefficient to adjust the relative weight of different zones, $\boldsymbol{S}_{D}\left(k, R_{b}\right)=$

$\left[\boldsymbol{S}^{(r, d)}\left(k, R_{b}\right)^{T}, \boldsymbol{S}^{(\theta, d)}\left(k, R_{b}\right)^{T}, \boldsymbol{S}^{(\phi, d)}\left(k, R_{b}\right)^{T}\right]^{T}$ is a $6(Q+1)^{2}$ long vector with

$$
\begin{aligned}
& \boldsymbol{S}^{(\Psi, d)}\left(k, R_{b}\right) \\
& \quad=\left[\mathcal{R}\left\{S_{00}^{(\Psi, d)}\right\}, \mathcal{I}\left\{S_{00}^{(\Psi, d)}\right\}, \ldots, \mathcal{R}\left\{S_{Q Q}^{(\Psi, d)}\right\}, \mathcal{I}\left\{S_{Q Q}^{(\Psi, d)}\right\}\right]^{T},
\end{aligned}
$$

where $\mathcal{R}\{\cdot\}$ denotes the real part, $\mathcal{I}\{\cdot\}$ denotes the imaginary part, $\boldsymbol{W}(k)=\left[\left|w_{1}(k)\right|^{2},\left|w_{2}(k)\right|^{2}, \ldots,\left|w_{N_{L}}(k)\right|^{2}\right]^{T}$ is a $N_{L}$ long vector, and $\boldsymbol{S}_{A}\left(k, R_{b}\right)=$

$$
\left[\boldsymbol{S}^{(r, a)}\left(k, R_{b}\right)^{T}, \boldsymbol{S}^{(\theta, a)}\left(k, R_{b}\right)^{T}, \boldsymbol{S}^{(\phi, a)}\left(k, R_{b}\right)^{T}\right]^{T} \text { is a }
$$
$6(Q+1)^{2}$ by $N_{L}$ matrix with

$$
\boldsymbol{S}^{(\Psi, a)}\left(k, R_{b}\right)=\left[\begin{array}{ccc}
\mathcal{R}\left\{S_{00}^{(\Psi, 1)}\right\} & \cdots & \mathcal{R}\left\{S_{00}^{\left(\Psi, N_{L}\right)}\right\} \\
\mathcal{I}\left\{S_{00}^{(\Psi, 1)}\right\} & \cdots & \mathcal{I}\left\{S_{00}^{\left(\Psi, N_{L}\right)}\right\} \\
\vdots & \ddots & \vdots \\
\mathcal{R}\left\{S_{Q Q}^{(\Psi, 1)}\right\} & \cdots & \mathcal{R}\left\{S_{Q Q}^{\left(\Psi, N_{L}\right)}\right\} \\
\mathcal{I}\left\{S_{Q Q}^{(\Psi, 1)}\right\} & \cdots & \mathcal{I}\left\{S_{Q Q}^{\left(\Psi, N_{L}\right)}\right\}
\end{array}\right]
$$

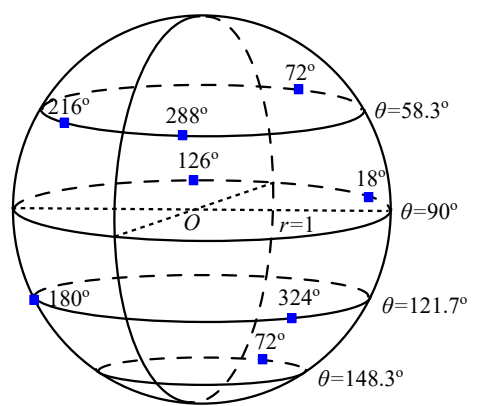

Fig. 2. The 8-channel loudspeaker array. The blue squares denote the loudspeakers.

$\boldsymbol{W} \geq 0$ means that each component of the vector $\boldsymbol{W}$ should be non-negative.

The optimization problem (7) can be rearranged as

$$
\min _{\boldsymbol{W}}\left\|\hat{\boldsymbol{S}}_{A} \boldsymbol{W}-\hat{\boldsymbol{S}}_{D}\right\|_{2}^{2}, \quad \text { s.t. } \boldsymbol{W} \geq 0,
$$

where $\hat{\boldsymbol{S}}_{A}=\left[\tau_{1} \boldsymbol{S}_{A}\left(k, R_{1}\right)^{T}, \ldots, \tau_{N_{P}} \boldsymbol{S}_{A}\left(k, R_{N_{P}}\right)^{T}\right]^{T}$, and $\hat{\boldsymbol{S}}_{D}=\left[\tau_{1} \boldsymbol{S}_{D}\left(k, R_{1}\right)^{T}, \ldots, \tau_{N_{P}} \boldsymbol{S}_{D}\left(k, R_{N_{P}}\right)^{T}\right]^{T}$. This problem is known as non-negative least squares (NNLS), and can be solved using the Lawson and Hanson's method [27]. Note that (10) can provide optimal sound intensity reproduction in each zone given an appropriate loudspeaker array, which allows for a non-uniform array arrangement.

\section{SIMULATIONS}

\subsection{Simulation setup and criteria}

In this simulation example, we simulate a rectangular room of size $(5,6,3) \mathrm{m}$, with the reflection coefficients of $\boldsymbol{d}=$ $(0.6,0.7,0.6,0.7,0.6,0.7)$. The image depth is $\boldsymbol{R}_{\text {depth }}=$ $(3,3,3)$. The loudspeaker array consists of eight loudspeakers non-uniformly distributed on a sphere with radius of $1 \mathrm{~m}$, as shown in Fig. 2. There are two 3D spherical zones (zone $\# 1$ and zone \#2) with radius $R_{1}=R_{2}=0.1 \mathrm{~m}$ inside the loudspeaker array, and their origins are located at $(0.3,0,0)$ $\mathrm{m}$ and $(-0.3,0,0) \mathrm{m}$, respectively. The desired soundfield is a plane wave coming from $(\pi / 3,4 \pi / 3)$ with frequency $f=900 \mathrm{~Hz}$ for both zones. We treat the two zones equally, i.e., $\tau_{1}=\tau_{2}=1$. Sound speed is $c=343 \mathrm{~m} / \mathrm{s}$ and air density is $\rho_{0}=1.29 \mathrm{~kg} / \mathrm{m}^{3}$. The mode matching method [13] is also implemented for comparison.

In [21], we have shown that accurate reconstruction of sound intensity guarantees a good localization perception within the target region by perceptual experiments. As the first objective performance measure, we define the relative error of sound intensity as

$$
\epsilon(k)=10 \log _{10}\left(\frac{\sum_{\forall \hat{\boldsymbol{x}}}\left|\mathcal{R}\left\{I_{\Psi}^{d}(\hat{\boldsymbol{x}}, k)-I_{\Psi}^{a}(\hat{\boldsymbol{x}}, k)\right\}\right|^{2}}{\sum_{\forall \hat{\boldsymbol{x}}}\left|\mathcal{R}\left\{I_{\Psi}^{d}(\hat{\boldsymbol{x}}, k)\right\}\right|^{2}}\right)(\mathrm{dB}),
$$

where $\hat{\boldsymbol{x}}$ denotes the evaluated point. The direction of intensity vector reflects the direction of travel of the sound, therefore, the intensity direction error $\eta$ is also defined to show the 


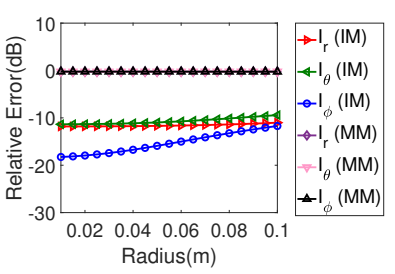

(a)

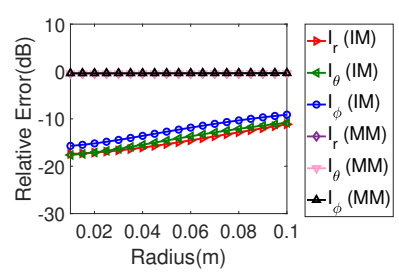

(b)
Fig. 3. Relative error with respect to the radius for (a) zone \#1 and (b) zone \#2 controlled by the intensity matching (IM) and the mode matching (MM).

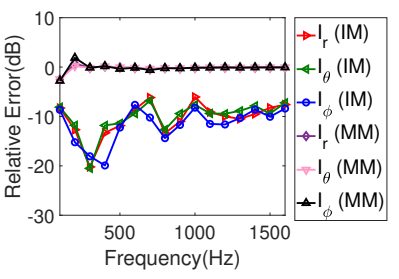

(a)

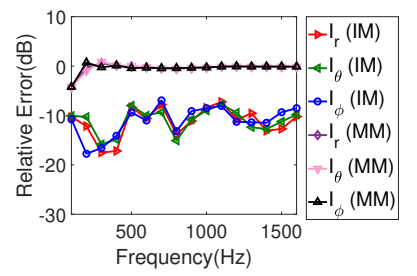

(b)
Fig. 4. Relative error on the surface of the target region with respect to the frequency for (a) zone \#1 and (b) zone \#2 controlled by IM and MM.

difference in angles between two vectors

$$
\eta(\hat{\boldsymbol{x}}, k)=\cos ^{-1}(\mathrm{DOT}) / \pi \times 100(\%),
$$

where

$$
\text { DOT }=\frac{\mathcal{R}\left\{\boldsymbol{I}^{a}(\hat{\boldsymbol{x}}, k)\right\}}{\left\|\mathcal{R}\left\{\boldsymbol{I}^{a}(\hat{\boldsymbol{x}}, k)\right\}\right\|_{2}} \cdot \frac{\mathcal{R}\left\{\boldsymbol{I}^{d}(\hat{\boldsymbol{x}}, k)\right\}}{\left\|\mathcal{R}\left\{\boldsymbol{I}^{d}(\hat{\boldsymbol{x}}, k)\right\}\right\|_{2}},
$$

with the desired intensity vector $\boldsymbol{I}^{d}(\hat{\boldsymbol{x}}, k)$ and the reproduced intensity vector $\boldsymbol{I}^{a}(\hat{\boldsymbol{x}}, k)$, respectively, at $\hat{\boldsymbol{x}}$. Note that here we only consider the real part of sound intensity which represents the propagating sound energy and shows the direction of propagation [21].

\subsection{Simulation results}

We first evaluate the reproduction of sound intensity within the two target zones for both the intensity matching method and the mode matching method. The results showing the relative error of sound intensity in the $r, \theta$, and $\phi$ directions are given in Fig. 3. The relative error controlled by the mode matching is around $0 \mathrm{~dB}$ for all the components of sound intensity vector within the two target zones. For the intensity matching, the relative error lies between $-20 \mathrm{~dB}$ and $-10 \mathrm{~dB}$ within the target zones, which is much better than the mode matching. We note that the relative error gradually increases for the intensity matching, whereas it remains for the mode matching, as the radius goes up.

To examine the performance of the proposed method with respect to the changes of frequencies, we calculate the relative error by varying the frequency between $100 \mathrm{~Hz}$ and $1600 \mathrm{~Hz}$. The observation sphere is the surface of each target zone (i.e., the spherical surface with the maximum error). The results

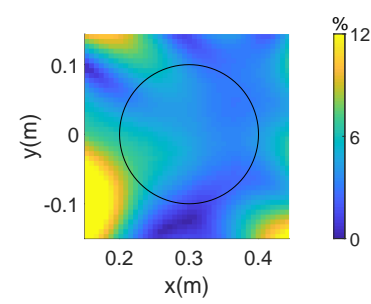

(a)

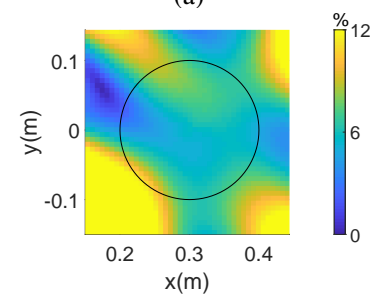

(c)

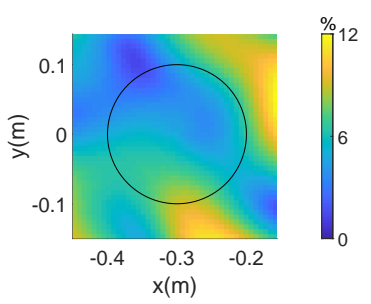

(b)

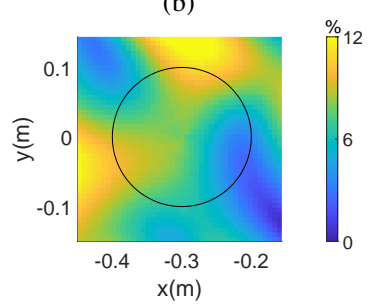

(d)
Fig. 5. The intensity direction error on plane $z=0$ for $(\mathrm{a}, \mathrm{c})$ zone \#1 and (b, d) zone \#2 controlled by (a-b) IM and (c-d) MM. Black circles denote the target zones.

are shown in Fig. 4. We observe that the proposed method has around $10 \mathrm{~dB}$ improvement compared with the mode matching for the evaluated frequency range in both zones. The different components of intensity vectors have similar curve fluctuation. This analysis proves that the proposed method can work for broadband multizone soundfield reproduction. Note that the big fluctuations along the frequency for the proposed method are due to the non-linear optimization.

The discussion above has shown that all the components of sound intensity can be reproduced with a limited error within the two target zones using the intensity matching method. However, it is clear that if we are concerned to create a realistic perception of the original sound, it requires to ensure the reproduction of the sound direction. Therefore, we calculate the intensity direction error on plane $z=0$ using (12). The results of the intensity matching are given in Fig. 5(a) and Fig. 5(b). The black circles denote the target zones in the figures. It shows that the intensity matching has less direction error within both the zones by comparing with the results of the mode matching in Fig. 5(c) and Fig. 5(d).

\section{CONCLUSION}

In this paper, we propose an intensity based reproduction method for multiple spatial zones in a reverberant room. We match sound intensity within the spatial zones by a cost function incorporating room transfer functions. We demonstrate, in the simulation example with a spatially non-uniform loudspeaker arrangement, that the proposed method can reproduce the desired sound intensity (both magnitude and direction) within the target zones with a smaller error than the conventional multizone reproduction method of mode matching, which may lead to better perception for listeners. Experimental validation of the proposed method will be conducted in future work. 


\section{REFERENCES}

[1] J.-W. Choi and Y.-H. Kim, "Generation of an acoustically bright zone with an illuminated region using multiple sources," J. Acoust. Soc. Amer., vol. 111, no. 4, pp. 1695-1700, 2002.

[2] S. J. Elliott, J. Cheer, J.-W. Choi, and Y. Kim, "Robustness and regularization of personal audio systems," IEEE Trans. Audio, Speech, Lang. Process., vol. 20, no. 7, pp. 2123-2133, 2012.

[3] P. Coleman, P. Jackson, M. Olik, M. Møller, M. Olsen, and J. A. Pederson, "Acoustic contrast, planarity and robustness of sound zone methods using a circular loudspeaker array," J. Acoust. Soc. Amer., vol. 135, no. 4, pp. 1929-1940, 2014.

[4] M. Poletti, "An investigation of 2-d multizone surround sound systems," in Proc. 125th Audio Eng. Soc. Conv., 2008.

[5] Nasim. Radmanesh and I. Burnett, "Generation of isolated wideband sound fields using a combined two-stage lasso-ls algorithm," IEEE Trans. Audio, Speech, Lang. Process., vol. 21, no. 2, pp. 378-387, 2012.

[6] J. H. Chang and F. Jacobsen, "Sound field control with a circular double-layer array of loudspeakers," J. Acoust. Soc. Amer., vol. 131, no. 6, pp. 4518-4525, 2012.

[7] M. F. S. Gálvez, S. J Elliott, and J. Cheer, "Time domain optimization of filters used in a loudspeaker array for personal audio," IEEE/ACM Trans. Audio, Speech, Lang. Process., vol. 23, no. 11, pp. 1869-1878, 2015.

[8] W. Zhang, T. D. Abhayapala, T. Betlehem, and F. M. Fazi, "Analysis and control of multi-zone sound field reproduction using modal-domain approach," J. Acoust. Soc. Amer., vol. 140, no. 3, pp. 2134-2144, 2016.

[9] W. Jin and W. B. Kleijn, "Theory and design of multizone soundfield reproduction using sparse methods," IEEE/ACM Trans. Audio, Speech, Lang. Process., vol. 23, no. 12, pp. 2343-2355, 2015.

[10] J. Donley, C. Ritz, and W. B. Kleijn, "Multizone soundfield reproduction with privacy-and quality-based speech masking filters," IEEE/ACM Trans. Audio, Speech, Lang. Process., vol. 26, no. 6, pp. 1041-1055, 2018.

[11] Z. Han, M. Wu, Q. Zhu, and J. Yang, "Twodimensional multizone sound field reproduction using a wave-domain method," J. Acoust. Soc. Amer., vol. 144, no. 3, pp. EL185-EL190, 2018.

[12] M. A. Poletti and F. M. Fazi, "An approach to generating two zones of silence with application to personal sound systems," J. Acoust. Soc. Amer., vol. 137, no. 2, pp. 598-605, 2015.

[13] Y. J. Wu and T. D. Abhayapala, "Spatial multizone soundfield reproduction: Theory and design," IEEE
Trans. Audio, Speech, Lang. Process., vol. 19, no. 6, pp. 1711-1720, 2010.

[14] M. Shin, F. M. Fazi, P. A. Nelson, and J. Seo, "Velocity controlled sound field reproduction by non-uniformly spaced loudspeakers," J. Sound Vib., vol. 370, pp. 444464, 2016.

[15] M. A. Gerzon, "Optimal reproduction matrices for multispeaker stereo," J. Audio Eng. Soc., vol. 40, no. 7/8, pp. 571-589, 1992.

[16] A. Ando and K. Hamasaki, "Sound intensity-based three-dimensional panning," in Proc. 126th Audio Eng. Soc. Conv. 2009.

[17] H. Hacihabiboğlu and Z. Cvetković, "Panoramic recording and reproduction of multichannel audio using a circular microphone array," in Proc. IEEE Workshop Appl. Signal Process. Audio Acoust., 2009, pp. 117-120.

[18] D. Arteaga, "An ambisonics decoder for irregular 3d loudspeaker arrays," in Proc. 134th Audio Eng. Soc. Conv., 2013.

[19] D. Scaini and D. Arteaga, "Decoding of higher order ambisonics to irregular periphonic loudspeaker arrays," in Proc. 55th Int. Audio Eng. Soc. Conf., 2014.

[20] M. Frank, Phantom sources using multiple loudspeakers in the horizontal plane, Ph.D. dissertation, 2013.

[21] H. Zuo, P. N. Samarasinghe, and T. D. Abhayapala, "Intensity based spatial soundfield reproduction using an irregular loudspeaker array," IEEE/ACM Trans. Audio, Speech, Lang. Process., vol. 28, pp. 1356-1369, 2020.

[22] H. Zuo, P. N. Samarasinghe, T. D. Abhayapala, and G. Dickins, "Spatial sound intensity vectors in spherical harmonic domain," J. Acoust. Soc. Amer., vol. 145, no. 2, pp. EL149-EL155, 2019.

[23] H. Zuo, P. N. Samarasinghe, T. D. Abhayapala, and G. Dickins, "Erratum: Spatial sound intensity vectors in spherical harmonic domain [j. acoust. soc. am. 145 (2), el149-el155 (2019)]," J. Acoust. Soc. Amer., vol. 146, no. 1, pp. 164-165, 2019.

[24] R. A. Kennedy, P. Sadeghi, T. D. Abhayapala, and H. M. Jones, "Intrinsic limits of dimensionality and richness in random multipath fields," IEEE Trans. Signal Process., vol. 55, no. 6, pp. 2542-2556, 2007.

[25] F. W. J. Olver, NIST Handbook of Mathematical Functions, Cambridge University Press, 2010.

[26] H. Zuo, T. D. Abhayapala, and P. N. Samarasinghe, "Particle velocity assisted three dimensional sound field reproduction using a modal-domain approach," IEEE/ACM Trans. Audio, Speech, Lang. Process., vol. 28, pp. 2119-2133, 2020.

[27] C. L. Lawson and R. J. Hanson, Solving least squares problems, vol. 15, Siam, 1995. 